\title{
Análise comparativa de comportamento mecânico de concreto reforçado com macrofibra polimérica e com fibra de aço
}

\author{
Comparative evaluation of the mechanical behavior \\ of synthetic and steel fiber-reinforced concrete
}

Renan Pícolo Salvador, Antonio Domingues de Figueiredo

\author{
Departamento de Engenharia de Construção Civil da Escola Politécnica \\ da Universidade de São Paulo, São Paulo, SP. \\ e-mail: renan.salvador@usp.br ; antonio.figueiredo@poli.usp.br
}

\begin{abstract}
RESUMO
A utilização de macrofibras poliméricas está amplamente difundida no mercado brasileiro. Entretanto, a caracterização do comportamento mecânico de concretos reforçados com essas macrofibras ainda deve ser mais bem avaliada. Dentre os diversos métodos utilizados para essa finalidade, os ensaios realizados com sistema fechado de controle de velocidade de deslocamento fornecem resultados mais confiáveis. A principal vantagem conferida por esse sistema está na avaliação desses compósitos em baixo nível de fissuração, com maior nível de acurácia. O desempenho desses compósitos foi estudado segundo uma norma recente (ASTM C1609 - 2010) e outra que é uma referência tradicional no Brasil (JSCE-SF4 - 1984). Ambos os procedimentos prescrevem ensaios de flexão de corpos-de-prova prismáticos para determinação da resistência residual pós-fissuração e da tenacidade. Foram estudadas duas fibras: uma macrofibra de polipropileno, nas dosagens de $0,22,0,33,0,50,0,66,0,82$ e 1,0\% em volume, e outra de aço, nas dosagens de $0,19,0,32$ e $0,45 \%$ em volume. Foi utilizada uma matriz de concreto com resistência média à compressão de 35MPa. Foi verificado que a macrofibra polimérica pode proporcionar o mesmo nível de resistência residual que a fibra de aço, desde que sejam determinados teores com equivalência de desempenho em estudo de dosagem. Além disso, foi comprovada a maior precisão do ensaio utilizando-se a velocidade de deslocamento do corpo-de-prova como parâmetro de controle do ensaio, devido à redução da instabilidade pós-pico, que propiciou melhor avaliação da resistência residual do compósito nos níveis iniciais de deslocamento e fissuração da matriz.
\end{abstract}

Palavras-chave: concreto reforçado com fibras, macrofibra sintética, tenacidade, sistema fechado de controle de deslocamento.

\section{ABSTRACT}

Synthetic macrofibers are commonly used in Brazilian market. However, the characterization of the mechanical behavior of reinforced concretes with these macrofibers still hast to be better evaluated. Among the many methods used to this purpose the tests performed under closed-loop displacement control provide more reliable results. The main advantage of that system is in a more accurate evaluation of these composites at low levels of crack opening. In this study, the performance of these composites was examined according to a recent standard (ASTM C1609 - 2010) and a more traditional one, which is reference in Brazil (JSCE-SF4 - 1984). Both procedures prescribe flexural tests in prismatic specimens for the determination of post-crack residual strength and toughness. Two fibers were analyzed: a polypropylene macrofiber, used in the dosages of $0.22,0.33,0.50,0.66,0.82$ and 1.0 in volume percentage, and a steel fiber, in the dosages of $0.19,0.32$ and 0.45 in volume percentage. A matrix of average compressive strength of $35 \mathrm{MPa}$ was used. It was observed that the synthetic macrofiber can provide the same level of residual strength as the steel fiber, as long as the equivalence of the dosages of each fiber is taken into account. Besides that, great efficiency and accuracy were obtained using the net displacement of the specimen as the controlled variable, due to the reduction of post-peak instability. Therefore, the determination of the residual strength of the composite in the initial levels of displacement and cracking of the matrix was better achieved.

Keywords: Fiber-reinforced concrete, Synthetic macrofiber, Toughness, Closed-loop control. 


\section{INTRODUÇÃO}

$\mathrm{O}$ concreto, material estrutural amplamente utilizado em todo o mundo, tem sido assunto de pesquisas contínuas no ramo da construção. Em parte, isso ocorre devido ao fato desse compósito apresentar certas limitações, como a baixa capacidade de deformação e a rápida propagação de fissuras quando submetido a esforços de tração [1, 2].

Com o intuito de minimizar tais limitações, o emprego de fibras para reforço de misturas cimentícias apresentou grande progresso nas últimas décadas. Diferentemente da armadura convencional, que é localizada e necessita de prévia montagem, as fibras são misturadas diretamente ao concreto e distribuídas aleatoriamente, reduzindo tempo, mão-de-obra e custo de certas aplicações em relação ao processo tradicional [1].

A incorporação de fibras em misturas cimentícias promove uma melhora significativa em diversas propriedades mecânicas, com destaque para a tenacidade à flexão e a resistência à fadiga e ao impacto. Sua principal função é aumentar a capacidade de absorção de energia, pois atuam como ponte de transferência de tensões através das fissuras, reduzindo sua propagação e expansão [3, 4]. Além disso, concreto reforçado com fibras apresenta maior ductilidade em relação às matrizes não reforçadas, que se tornam deficientes após a formação de primeira fissura $[5,6]$.

Diversos estudos foram publicados sobre a investigação do comportamento de fibras de aço em matrizes de concreto [2, 4-8]. As reações ocasionadas por esforços de flexão e a deformação resultante desse processo são caracterizadas. Os parâmetros fundamentais que influenciam as propriedades do concreto reforçado com fibras são o tipo de fibra (material constituinte, geometria e propriedades de interface com a matriz) e sua fração volumétrica [7, 9].

A forma mais tradicional para a avaliação do comportamento do concreto com fibras é por meio da determinação da tenacidade ou da resistência residual pós-fissuração no ensaio de flexão em prismas. Existem várias normas que focam esse tipo de ensaio, como a ASTM C1609-2010 [10], a ASTM C13992010 [11], a JSCE-SF4-1984 [12] e a EN 14651-2007 [13]. Ensaios alternativos a essa configuração, como é o caso do Ensaio Barcelona [14], uma modificação do ensaio de puncionamento duplo, podem ser utilizados.

O parâmetro de controle da velocidade do ensaio apresenta grande influência na obtenção da curva carga-deslocamento vertical [15]. Quando são utilizados sistemas open-loop, nos quais a velocidade do pistão é utilizada para controlar o ensaio, é frequente a ocorrência de uma região de instabilidade pós-pico. No momento em que a matriz atinge sua carga máxima e fissura, a carga é transferida para as fibras de forma abrupta, ocorrendo uma instabilidade [16]. Este fenômeno é característico de concretos com baixo teor de fibras, pois a capacidade resistente pós-fissuração é menor que a capacidade resistente da matriz. Seu efeito é uma superestimativa da deformação do material, aumentando a área abaixo da curva para baixos níveis de deslocamento e, consequentemente, a tenacidade do compósito [16].

O método mais preciso para determinação da tenacidade e da resistência residual pós-fissuração de matrizes cimentícias reforçadas com fibras é baseado em um sistema fechado de controle de velocidade de deslocamento (closed-loop). Utilizando esse sistema, obtém-se uma grande diminuição da instabilidade após rompimento da matriz cimentícia, conferindo maior confiabilidade à caracterização de diferentes fibras para reforço de concreto $[15,16]$. Como fator complementar que auxilia na precisão do ensaio, é necessário utilizar equipamentos de alta rigidez para que, após o rompimento da matriz, a energia não seja dissipada no corpo-de-prova em teste.

Apesar destes fatos já serem discutidos na literatura há algum tempo, não se tem conhecimento de resultados publicados utilizando-se essa metodologia no Brasil, para a avaliação de desempenho de macrofibras poliméricas. Além disso, poucos são os resultados disponíveis sobre a avaliação de concretos reforçados com essas fibras, que consistem em uma alternativa tecnológica mais recente e pouco enfocada em pesquisas [17]. Dessa forma, no sentido de prover valores referenciais de comportamento para esse tipo de compósito nesta condição de avaliação, foi desenvolvido o estudo experimental descrito a seguir.

\section{MATERIAIS E MÉTODOS}

Para avaliar o comportamento mecânico do concreto reforçado com fibras, os procedimentos utilizados foram os propostos pela norma ASTM C1609 (2010) e pelo método JSCE-SF4 (1984). O primeiro procedimento é mais recente e prescreve a utilização de sistema fechado de controle de velocidade de deslocamento. Já o segundo é um dos métodos mais utilizados para o dimensionamento de concreto com fibras, além de ser o mais amplamente utilizado no Brasil. 
Foram ensaiados corpos-de-prova prismáticos de dimensões 150x150x500 $\mathrm{mm}^{3}$, com vão de ensaio de $450 \mathrm{~mm}$. A carga é aplicada por dois cutelos superiores, apoiados sobre o terço central do vão do prisma, e é controlada pelo deslocamento vertical do corpo-de-prova. Foram utilizadas duas velocidades de aplicação de carga: no primeiro trecho, correspondente ao deslocamento de 0 a $0,75 \mathrm{~mm}$, a velocidade foi de $0,12 \mathrm{~mm} / \mathrm{min}$. No segundo, entre 0,75 e $3,0 \mathrm{~mm}$, a velocidade foi de $0,24 \mathrm{~mm} / \mathrm{min}$. É adotada uma menor velocidade no trecho inicial, pois este é a etapa crítica da realização dos ensaios, quando a carga é transferida da matriz para as fibras. Esse intervalo de deslocamento está associado ao trecho elástico ( 0 a $0,05 \mathrm{~mm}$ de deslocamento, aproximadamente) e ao início do comportamento elasto-plástico. No segundo trecho, correspondente ao comportamento pós-fissuração, foi adotada uma velocidade maior de ensaio, pois não é esperado nenhum tipo de instabilidade nesta etapa.

Foram avaliadas duas fibras para reforço de concreto, uma polimérica e uma de aço, de modo a possibilitar a avaliação comparativa de desempenho desses dois tipos de fibras. Foi utilizada uma matriz de concreto de resistência média à compressão de $35 \mathrm{MPa}$, com dosagens de 0,22, 0,33, 0,50, 0,66, 0,82 e 1,0\% em volume para a macrofibra sintética; e de 0,19,0,32 e 0,45\% em volume para a fibra de aço. $\mathrm{O}$ procedimento de moldagem foi realizado segundo o método JSCE-SF2 [18]. Para cada traço, foram moldados 10 corpos-de-prova prismáticos para ensaio de flexão e 6 cilíndricos, de dimensões $150 \times 300 \mathrm{~mm}^{2}$ (diâmetro x altura) para ensaio de compressão axial, segundo ABNT 5739 (2007) [19].

As Tabelas 1 e 2 apresentam os consumos, a composição e as características do traço de concreto utilizado no estudo. As propriedades das fibras utilizadas nos ensaios estão listadas na Tabela 3. O valor especificado para o abatimento é o de referência para a matriz sem a adição das fibras. Neste estudo, optou-se por elevar este valor a $120 \mathrm{~mm}$ e verificar o impacto da utilização da fibra no abatimento do concreto. Este abatimento não foi corrigido pelo fato de se utilizar baixos volumes de fibra, o que não proporciona grande prejuízo à compactação quando o material é vibrado [20].

Tabela 1: Consumos dos materiais para a produção do concreto utilizado no estudo.

\begin{tabular}{l|l}
\hline MATERIAL & DOSAGEM $\left(\mathrm{Kg} / \mathbf{m}^{3}\right)$ \\
\hline Cimento CPV ARI & 330,00 \\
\hline Agregado miúdo & 825,00 \\
\hline Agregado graúdo & 1023,00 \\
\hline Água & 198,00 \\
\hline Aditivo polifuncional & 1,32 \\
\hline
\end{tabular}

Tabela 2: Traço e características do concreto.

\begin{tabular}{l|l}
\hline Traço unitário & $1,00: 2,50: 3,10: 0,60$ \\
\hline Massa específica / kg/m $\mathrm{m}^{3}$ & 2287 \\
\hline Ar incorporado / \% & 3,2 \\
\hline Abatimento / mm & 120 \\
\hline Teor de argamassa seca / \% & 53,0 \\
\hline $\mathrm{f}_{\mathrm{cm}} / \mathrm{MPa}$ & 35 \\
\hline
\end{tabular}


Tabela 3: Propriedades das fibras utilizadas.

\begin{tabular}{l|l|l}
\hline PROPRIEDADE & MACROFIBRA SINTÉTICA & FIBRA DE AÇO \\
\hline Material & copolímero de polipropileno virgem & aço \\
\hline Forma & monofilamento / fibrilado & Tipo A1 [21] \\
\hline $\begin{array}{l}\text { Número de filamentos por } \\
\text { quilograma }\end{array}$ & 221.000 & 4.600 \\
\hline Comprimento / mm & 54 & 60 \\
\hline Diâmetro equivalente / mm & 0,32 & 0,75 \\
\hline Massa específica / g/cm 3 & 0,91 & 7,85 \\
\hline Módulo de elasticidade / GPa & 5 & 210 \\
\hline Resistência à tração / MPa & $570-660$ & 1100 \\
\hline
\end{tabular}

Para a análise da curva carga-deslocamento vertical segundo a norma ASTM C1609, são calculadas as resistências residuais em 0,75 e 3,0mm de deslocamento ( $\mathrm{f}_{150 ; 0.75}$ e $\mathrm{f}_{150 ; 3.0}$, respectivamente), utilizando as seguintes equações:

$$
\mathrm{f}_{150 ; 0.75}=\mathrm{P}_{150 ; 0.75} \times\left(\mathrm{L} / \mathrm{b} . \mathrm{d}^{2}\right)
$$

$$
\mathrm{f}_{150 ; 3.0=} \mathrm{P}_{150 ; 3.0} \mathrm{x}\left(\mathrm{L} / \mathrm{b} . \mathrm{d}^{2}\right)
$$

nas quais

$\mathrm{f}_{150 ; 0.75}$ e $\mathrm{f}_{150 ; 3.0}$ : resistências residuais quando o deslocamento vertical do corpo-de-prova for 0,75 e $3,0 \mathrm{~mm}$, respectivamente $(\mathrm{MPa})$;

$\mathrm{P}_{150 ; 0.75}$ e $\mathrm{P}_{150 ; 3.0}$ : cargas residuais quando o deslocamento vertical do corpo-de-prova for 0,75 e 3,0mm, respectivamente $(\mathrm{N})$;

L: vão de ensaio (mm);

b: largura do corpo-de-prova (mm);

d: altura do corpo-de-prova ( $\mathrm{mm})$.

Para analisar as curvas segundo o método JSCE-SF4, é calculado o fator de tenacidade à flexão segundo a equação:

$$
\overline{\sigma_{\mathrm{b}}}=\left(\mathrm{T}_{\mathrm{b}} / \delta_{\mathrm{tb}}\right) \cdot\left(\mathrm{L} / \mathrm{b} \cdot \mathrm{d}^{2}\right)
$$

na qual

$\bar{\sigma}_{\mathrm{b}}$ : Fator de tenacidade à flexão quando o deslocamento vertical do corpo-de-prova for 3,0mm (MPa); $(\mathrm{J})$;

$\mathrm{T}_{\mathrm{b}}$ : Tenacidade à flexão (área abaixo da curva carga por deslocamento vertical), no intervalo de $0 \mathrm{a} \delta_{\mathrm{tb}}$

$\delta_{\mathrm{tb}}$ : Deslocamento vertical no ponto L/150 mm (como são utilizados corpos-de-prova com 450mm de vão de ensaio, $\left.\delta_{\mathrm{tb}}=3,0 \mathrm{~mm}\right)$;

L: Vão de ensaio (mm);

b: Largura do corpo-de-prova ( $\mathrm{mm})$;

d: Altura do corpo-de-prova (mm). 


\section{RESULTADOS E DISCUSSÃO}

\subsection{Propriedades do estado fresco}

As propriedades do estado fresco têm grande influência nos processos de moldagem e nas propriedades do estado endurecido. Por esse motivo, é importante que elas sejam uniformes em todos os traços utilizados. As propriedades medidas estão mostradas na Tabela 4. Como pode ser visto, houve uma redução do abatimento com o aumento do teor de fibra. Isto ocorre pelo fato da fibra prejudicar a mobilidade dos agregados e, consequentemente, reduzir a fluidez do material para baixos níveis de taxa de cisalhamento.

No entanto, como demonstrado por Ceccato e Figueiredo (1999) [20], uma vez utilizada, a vibração recupera boa parte da mobilidade e da capacidade de compactação do material quando baixos teores de fibras (abaixo de $1 \%$ em volume) são utilizados. Isto é corroborado pela uniformidade dos resultados de ar incorporado e pela baixíssima variação entre os lotes produzidos em termos de resistência à compressão, o que é mais bem analisado no próximo item. Isto garantiu as condições de comparação dos diferentes compósitos.

Tabela 4: Propriedades do concreto em estado fresco.

\begin{tabular}{l|l|l|l|l}
\hline TRAÇO & $\begin{array}{l}\text { DOSAGEM / \% } \\
\text { EM VOLUME }\end{array}$ & $\begin{array}{l}\text { ABATIMENTO / MM } \\
\text { (NM 67:1998) [22] }\end{array}$ & $\begin{array}{l}\text { MASSA ESPECÍFICA / Kg/m } \\
\text { (NBR 9833:2008) [23] }\end{array}$ & $\begin{array}{l}\text { AR INCORPORADO / } \\
\text { \% (NM 47:2002) [24] }\end{array}$ \\
\hline $\begin{array}{l}\text { Concreto } \\
\text { simples }\end{array}$ & 0 & 120 & 2.287 & 3,2 \\
\hline \multirow{2}{*}{$\begin{array}{l}\text { Macrofibra } \\
\text { polimérica }\end{array}$} & 0,22 & 75 & 2.288 & 3,2 \\
\cline { 2 - 5 } & 0,33 & 65 & 2.311 & 3,0 \\
\cline { 2 - 5 } & 0,50 & 50 & 2.278 & 3,2 \\
\cline { 2 - 5 } & 0,66 & 30 & 2.300 & 2,8 \\
\cline { 2 - 5 } & 0,82 & 25 & 2.311 & 1,0 \\
\cline { 2 - 5 } & 1,0 & 35 & 2.315 & 2,2 \\
\hline \multirow{2}{*}{ Fibra de aço } & 0,19 & 55 & 2.317 & 2,7 \\
\cline { 2 - 5 } & 0,32 & 80 & 2.227 & 3,4 \\
\cline { 2 - 5 } & 0,45 & 50 & 2.330 & 2,4 \\
\hline
\end{tabular}

\subsection{Resistência à compressão}

Para cada traço, foram moldados 6 corpos-de-prova cilíndricos de $150 \mathrm{~mm}$ de diâmetro para o ensaio de resistência à compressão. Os resultados estão mostrados na tabela 5 e, de acordo com os dados obtidos, verifica-se que houve baixa variação entre eles . Com os traços utilizados, a resistência à compressão é praticamente independente do teor de fibra. Tal fato já era esperado e corrobora com dados presentes na literatura $[25-\underline{28]}$. A resistência à compressão média entre todos os ensaios foi de 36,7MPa, com coeficiente de variação de 4,0\%. Assim, atingiu-se a resistência média prevista para este programa experimental. Com a fibra de aço, houve maior desvio padrão dentro do ensaio devido à maior dificuldade de moldagem dos corpos-de-prova com a fibra rígida.

\subsection{Ensaio de flexão}

Para cada traço, foram moldados 10 corpos-de-prova prismáticos de $150 \times 150 \times 500 \mathrm{~mm}^{3}$ para o ensaio de flexão. Para cada corpo-de-prova, foi obtida uma curva de carga por deslocamento vertical, e para cada conjunto de corpos-de-prova do mesmo traço, foi calculado seu valor médio. A Figura 1 apresenta as curvas para a macrofibra polimérica nas dosagens de 0,22 e de $1,0 \%$ e na Figura 2 encontram-se as curvas para a fibra de aço nas dosagens de 0,19 e $0,45 \%$. Percebe-se claramente que houve uma baixa variabilidade nos resultados e o comportamento apresentado nas curvas é bem uniforme, sem os sinais de instabilidade póspico. Na Figura 3 estão apresentadas as curvas médias de carga por deslocamento vertical dos concretos reforçados com as duas fibras. Percebe-se claramente que as fibras acabam por alterar a resistência residual pós-fissuração, com pouca influência na carga de pico, como era esperado, dado que se está trabalhando abaixo do volume crítico de fibras [1]. 
Tabela 5: Resultados de resistência à compressão.

\begin{tabular}{l|l|l}
\hline \multirow{4}{*}{ FIBRA } & DOSAGEM / \% EM VOLUME & FCM / MPA \\
\hline \multirow{4}{*}{$\begin{array}{l}\text { Macrofibra } \\
\text { polimérica }\end{array}$} & 0,22 & $38,32 \pm 0,94$ \\
\cline { 2 - 3 } & 0,33 & $37,68 \pm 0,50$ \\
\cline { 2 - 3 } & 0,50 & $34,33 \pm 0,18$ \\
\cline { 2 - 3 } & 0,66 & $34,85 \pm 0,24$ \\
\cline { 2 - 3 } & 0,82 & $36,77 \pm 0,44$ \\
\hline \multirow{4}{*}{ Fibra de aço } & 0,0 & $36,57 \pm 0,36$ \\
\cline { 2 - 3 } & 0,32 & $38,78 \pm 0,60$ \\
\cline { 2 - 3 } & 0,45 & $36,12 \pm 0,98$ \\
\hline
\end{tabular}

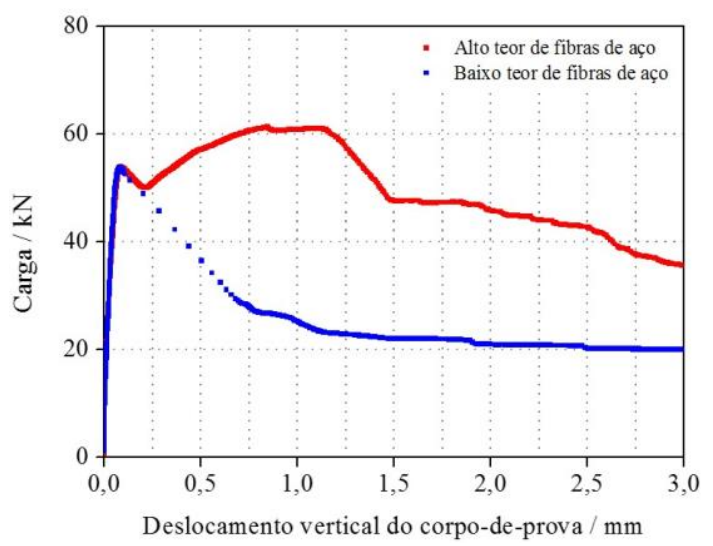

Figura 1: Curvas de carga por deslocamento vertical obtidas com o concreto reforçado com a macrofibra polimérica nas dosagens de $0,22 \%$ (a) e de $1,0 \%$ (b).
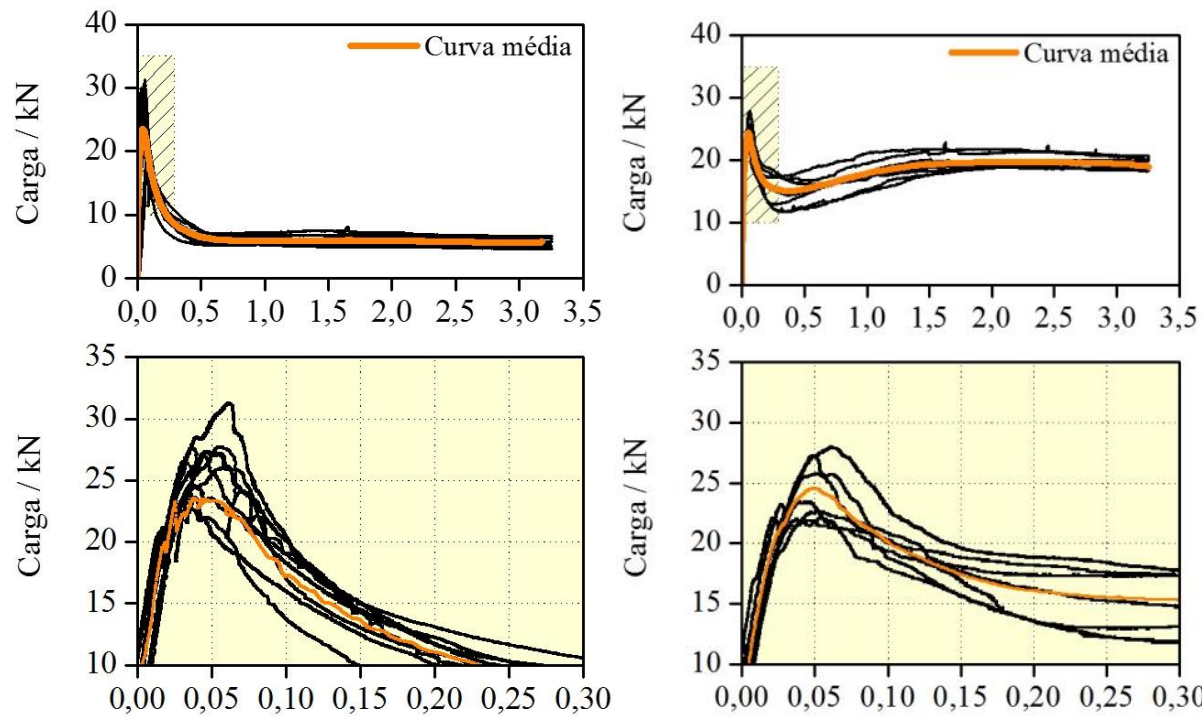

Deslocamento vertical do corpo-de-prova / mm

Deslocamento vertical do corpo-de-prova / $\mathrm{mm}$ 
Figura 2: Curvas de carga por deslocamento vertical obtidas com o concreto reforçado com a fibra de aço nas dosagens de $0,19 \%$ (a) e de $0,45 \%$ (b). Observação: em (a) há apenas 5 curvas de carga por deslocamento vertical, pois foram descartados 5 corpos-de-prova desse traço devido a rompimentos que ocorreram fora do terço central do vão de ensaio.
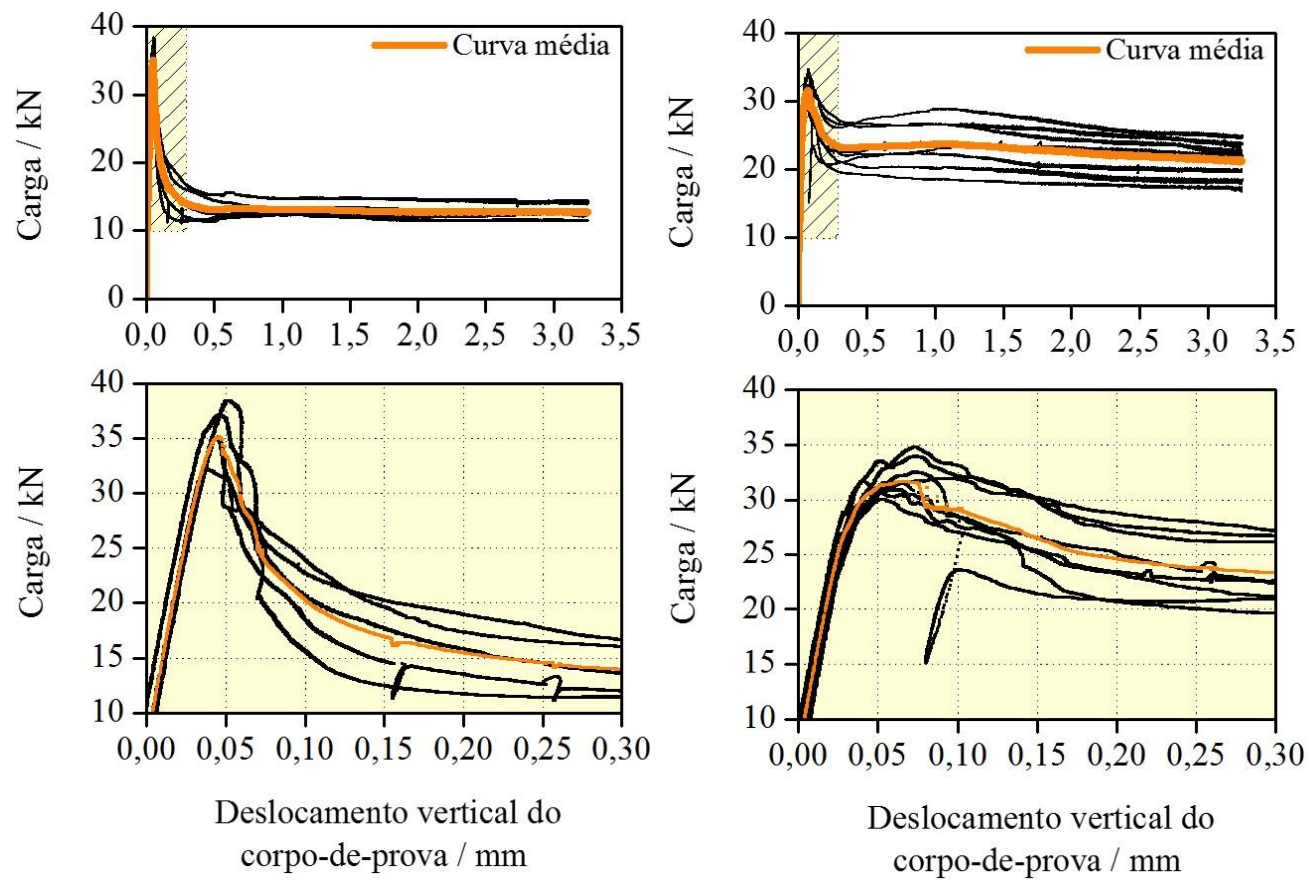

Figura 3: Curvas médias de carga por deslocamento vertical obtidas com o concreto reforçado com a macrofibra polimérica (a) e com a fibra de aço (b).

Em cada gráfico da Figura 3, verifica-se que a resposta elástica inicial, compreendida no intervalo entre 0 e $0,04 \mathrm{~mm}$ de deslocamento vertical, é praticamente inalterada pela adição das fibras, independentemente da dosagem utilizada [1]. O trecho entre o início da aplicação de carga e o ponto onde a carga máxima é atingida define o módulo de elasticidade do compósito, que é devido às características da matriz cimentícia, sem adição de fibras.

A carga resistida pós-fissuração aumenta com o consumo de fibras e é dependente do módulo de elasticidade da fibra e da sua aderência à matriz. Portanto, a resposta pós-fissuração de concretos reforçados com macrofibras poliméricas ou com fibras de aço tende a ser bastante distinta [26]. Em todos os casos, a ruptura ocorreu devido a apenas uma fissura discreta (não foi verificada a formação de múltiplas fissuras).

No caso das fibras de aço, a ancoragem mecânica na matriz é controlada principalmente pela aderência entre a fibra e a matriz e pelas deformações que existem em suas extremidades. As fibras, quando utilizadas em teores abaixo do volume crítico, sofrem um deslizamento logo que a carga é transferida para elas. Nesse momento, apresentam capacidade resistente inferior à carga resistida pela matriz no momento de sua fissuração, o que explica o comportamento de amolecimento de deslocamento (deflection-softening). A recuperação da capacidade de absorção de energia é alcançada quando a ancoragem é mobilizada [26, 27]. Este ganho de resistência residual, no entanto, é mais intenso para os menores níveis de deformação [29].

Para a macrofibra polimérica, a queda inicial na absorção de energia é mais pronunciada e ocorre em um intervalo de deslocamento maior, devido à menor rigidez das fibras poliméricas. Como o rompimento da matriz ocorre com pequenas deformações, as fibras poliméricas têm menor capacidade de absorção de energia para baixas deflexões, quando comparadas às fibras de aço [1]. Entretanto, para grandes aberturas de fissuras, o comportamento tende a ser o oposto, ou seja, a energia absorvida pelas fibras poliméricas pode superar a absorvida pelas fibras de aço [26].

Nos rompimentos realizados com o concreto reforçado com a macrofibra polimérica, verifica-se que em dosagens superiores a $0,50 \%$ em volume, a energia absorvida foi aumentando com o decorrer do ensaio. Essa resposta pode ser considerada como o encruamento da fibra [26, 27, 30], mas não do compósito.

Tratando-se especificamente do momento que sucede o rompimento da matriz, verifica-se que não ocorreu instabilidade, mesmo para as menores dosagens de fibra. Devido à utilização do sistema fechado de 
controle de deformação, o deslocamento abrupto do corpo-de-prova e, consequentemente, o do LVDT, foi evitado. Portanto, a curva obtida pode ser considerada como uma resposta mais precisa e estável, pois a resistência residual apresentada pelo compósito imediatamente após a carga de pico não está deturpada pela instabilidade do equipamento durante o procedimento do ensaio [15].

A partir da análise das curvas experimentais, foram calculados a resistência residual em 0,75 e $3,0 \mathrm{~mm}$ de deslocamento vertical e o fator de tenacidade à flexão. Os resultados estão apresentados na Tabela 6. Chama a atenção o nível muito baixo de variação dos resultados, que ficaram bem abaixo dos $30 \%$ de coeficiente de variação apresentados pela literatura como esperados para este tipo de ensaio [31].

Tabela 6: Fator de tenacidade à flexão e resistências residuais em 0,75 e em 3,0mm de deslocamento vertical, calculados a partir das curvas de carga por deslocamento vertical de cada corpo-de-prova de cada traço.

\begin{tabular}{|c|c|c|c|c|}
\hline FIBRA & $\begin{array}{l}\text { DOSAGEM / \% } \\
\text { EM VOLUME }\end{array}$ & $\begin{array}{l}\text { RESISTÊNCIA RESIDUAL } \\
\text { (F150;0.75) / MPA }\end{array}$ & $\begin{array}{l}\text { RESISTÊNCIA RESIDUAL } \\
\text { (F150;3.0) / MPA }\end{array}$ & $\begin{array}{l}\text { FATOR DE } \\
\text { TENACIDADE À } \\
\text { FLEXÃO }\left(\overline{\sigma_{b}}\right) / \text { MPA }\end{array}$ \\
\hline \multirow{6}{*}{$\begin{array}{l}\text { Macrofibra } \\
\text { polimérica }\end{array}$} & 0,22 & $0,800 \pm 0,078$ & $0,750 \pm 0,093$ & $0,918 \pm 0,075$ \\
\hline & 0,33 & $1,20 \pm 0,08$ & $1,22 \pm 0,10$ & $1,32 \pm 0,08$ \\
\hline & 0,50 & $1,58 \pm 0,19$ & $1,77 \pm 0,13$ & $1,81 \pm 0,14$ \\
\hline & 0,66 & $1,88 \pm 0,10$ & $2,00 \pm 0,16$ & $2,01 \pm 0,10$ \\
\hline & 0,82 & $2,02 \pm 0,15$ & $2,25 \pm 0,21$ & $2,17 \pm 0,17$ \\
\hline & 1,0 & $2,17 \pm 0,26$ & $2,60 \pm 0,12$ & $2,45 \pm 0,18$ \\
\hline \multirow{3}{*}{$\begin{array}{l}\text { Fibra de } \\
\text { aço }\end{array}$} & 0,19 & $1,76 \pm 0,16$ & $1,70 \pm 0,13$ & $1,81 \pm 0,14$ \\
\hline & 0,32 & $2,38 \pm 0,28$ & $2,23 \pm 0,24$ & $2,37 \pm 0,24$ \\
\hline & 0,45 & $3,12 \pm 0,43$ & $2,86 \pm 0,35$ & $3,08 \pm 0,40$ \\
\hline
\end{tabular}

De acordo com os dados apresentados na tabela 6, verifica-se que para as fibras de aço, a resistência residual em $0,75 \mathrm{~mm}$, considerada como a resistência do estado limite de serviço do compósito [27], é maior do que a resistência residual em $3,0 \mathrm{~mm}$, pois, para maiores deflexões, a fibra já apresentou considerável grau de escorregamento.

Entretanto, para a macrofibra polimérica nas dosagens de 0,22 e $0,33 \%$ em volume, a resistência residual nos dois níveis de deflexão é praticamente a mesma. Para as demais dosagens, a resistência residual em $3,0 \mathrm{~mm}$ supera a medida em $0,75 \mathrm{~mm}$, devido ao encruamento da fibra. A diferença entre os dois valores pode chegar a $20 \%$ quando o teor de fibra é de $1,0 \%$.

Comparando-se o parâmetro de controle da ASTM C1609 com o da JSCE-SF4, verifica-se que o valor do fator de tenacidade é mais alto do que o valor da resistência residual para a macrofibra polimérica em baixas dosagens. Isso se deve ao fato da área de pico ser considerada no cálculo da tenacidade pelo método japonês, apresentando maior influência quando a resposta pós-fissuração é mais baixa (menor área sob a curva). Entretanto, à medida que o teor de fibra é aumentado, o valor da resistência residual é mais alto do que o fator de tenacidade, pois, como o encruamento da fibra (slip-hardening) é mais pronunciado, o valor da carga no ponto de deslocamento de $3,0 \mathrm{~mm}$ é maior do que a carga média da curva.

Como a densidade do aço $\left(7,85 \mathrm{~g} / \mathrm{cm}^{3}\right)$ é cerca de 9 vezes maior que a do polipropileno $\left(0,91 \mathrm{~g} / \mathrm{cm}^{3}\right)$, a quantidade de fibras de aço por unidade de massa é pequena. Além disso, sua dispersão no concreto e o processo de moldagem são mais difíceis, devido à sua alta rigidez. Com isso, o número de fibras de aço na seção de ruptura não é homogêneo em todos os corpos-de-prova, conduzindo à maior dispersão nos resultados.

Os resultados apresentados na Tabela 6 foram utilizados para a produção de gráficos de correlação entre a medida da tenacidade ou da resistência residual e o teor de fibra. Foram obtidos os gráficos de dosagem das fibras, apresentados na Figura 4, que permitem determinar o teor de fibra que corresponde à demanda de desempenho média avaliada pelo ensaio de flexão em prismas com deslocamento controlado. 


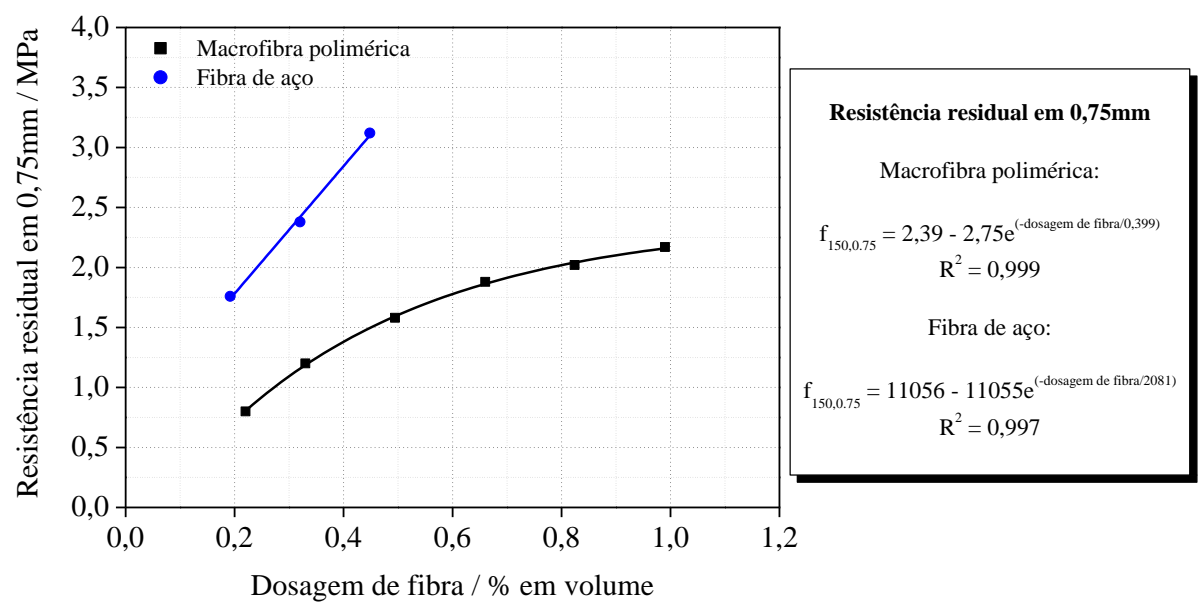

(a)

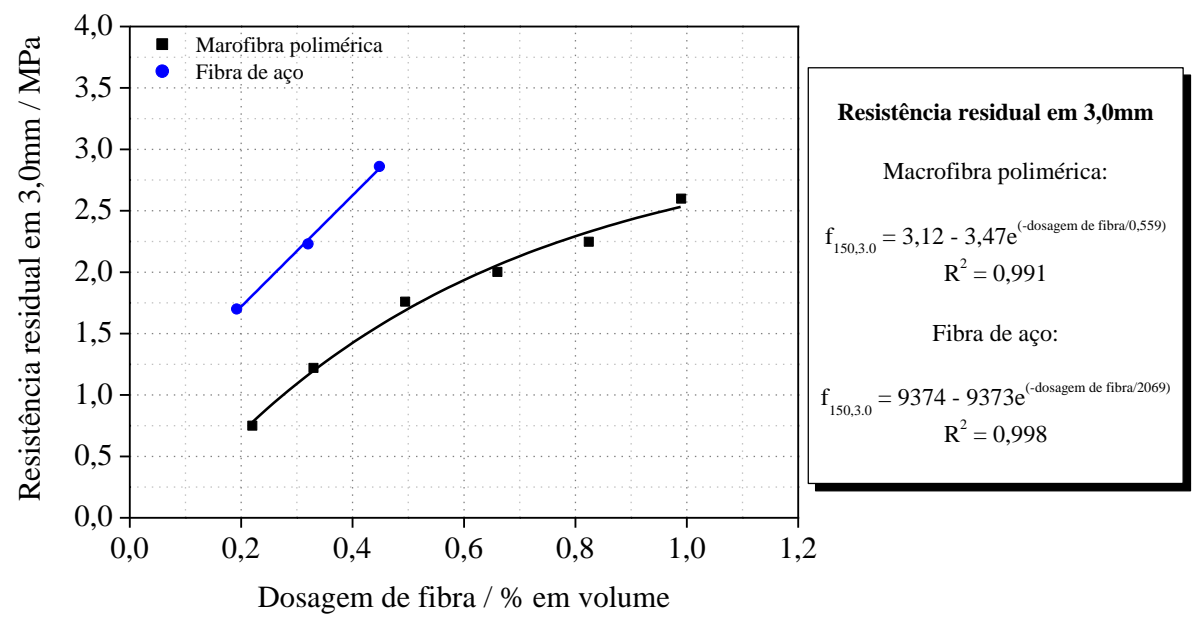

(b)

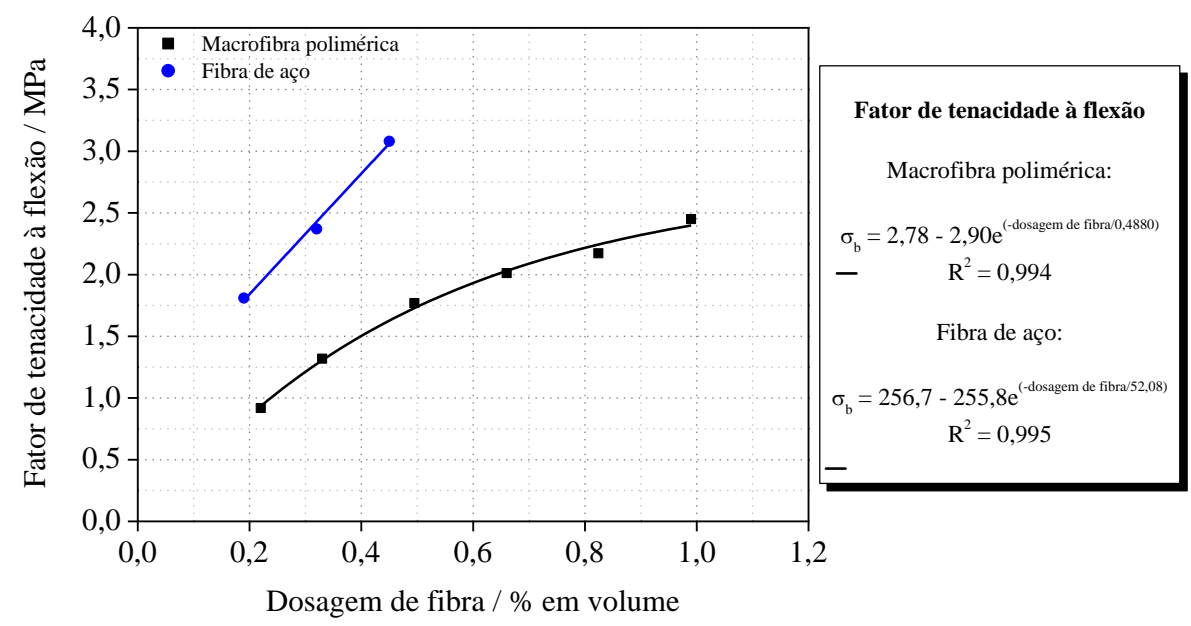

(c)

Figura 4: Correlação entre a tensão residual e o teor de fibras: (a) resistência residual em $0,75 \mathrm{~mm}$; (b) resistência residual em 3,0mm; (c) fator de tenacidade à flexão.

Nos três gráficos da Figura 4, foi obtida excelente aderência da curva de tendência aos dados experimentais, visto que o valor de $\mathrm{R}^{2}$ foi superior a $0,99 \mathrm{em}$ todas as regressões. Como esperado, quando se 
compara um mesmo teor em volume de fibras de aço e poliméricas, as fibras de aço conferem maiores resistências residuais, principalmente para baixos níveis de deformação, devido à sua maior rigidez.

O comportamento assintótico pode ser explicado pelo modelo proposto por ARMELIN e B ANTHIA [32], que estudaram o comportamento do concreto reforçado com fibras de aço pelo ensaio de single pullout das fibras. Segundo esses autores, com o aumento do consumo de fibras, há um maior gasto energético pela deformação plástica da parcela comprimida da seção flexionada no ensaio de determinação da tenacidade. Essa energia representa uma perda em termos de energia absorvida no corpo-de-prova e, consequentemente, da tenacidade. Tal fato foi também observado por FIGUEIREDO, et al. [33].

Para a avaliação comparativa de desempenho, as equações mostradas na figura 4 foram resolvidas adotando-se valores fixos para as tensões residuais. Como as equações obtidas com a macrofibra polimérica foram funções exponenciais, as correlações foram transformadas para a escala logarítmica, de modo a transformá-las em funções lineares, para facilitar os cálculos. Os resultados encontram-se na Tabela 7.

Tabela 7: Análise comparativa de dosagens de macrofibra e de fibra de aço para as tensões residuais.

\begin{tabular}{l|r|r|r}
\hline \multirow{2}{*}{$\begin{array}{l}\text { TENSÃO } \\
\text { RESIDUAL }\end{array}$} & VALOR / MPA & $\begin{array}{l}\text { DOSAGEM DE FIBRA POLIMÉRICA } \\
\text { \% EM VOLUME }\end{array}$ & $\begin{array}{l}\text { loSAGEM DE FIBRA DE AÇO } \\
\text { \% EM VOLUME }\end{array}$ \\
\hline \multirow{2}{*}{$\mathrm{f}_{150,0.75}$} & 1,80 & 0,638 & 0,203 \\
\cline { 2 - 5 } & 1,90 & 0,711 & 0,222 \\
\cline { 2 - 5 } & 2,00 & 0,794 & 0,241 \\
\hline \multirow{2}{*}{$\mathrm{f}_{150,3.0}$} & 1,80 & 0,534 & 0,218 \\
\cline { 2 - 5 } & 2,00 & 0,633 & 0,262 \\
\cline { 2 - 5 } & 2,20 & 0,749 & 0,306 \\
\cline { 2 - 5 } & 2,40 & 0,886 & 0,350 \\
\hline \multirow{2}{*}{$\sigma_{\mathrm{b}}$} & 2,80 & 0,532 & 0,193 \\
\cline { 2 - 5 } & 2,00 & 0,651 & 0,234 \\
\cline { 2 - 5 } & 2,40 & 0,796 & 0,275 \\
\hline
\end{tabular}

Os resultados apresentados na Tabela 7 foram utilizados para a obtenção da correlação entre as dosagens de macrofibra e de fibra de aço para as mesmas demandas de desempenho. As equações foram obtidas por regressões lineares e estão mostradas na Figura 5.

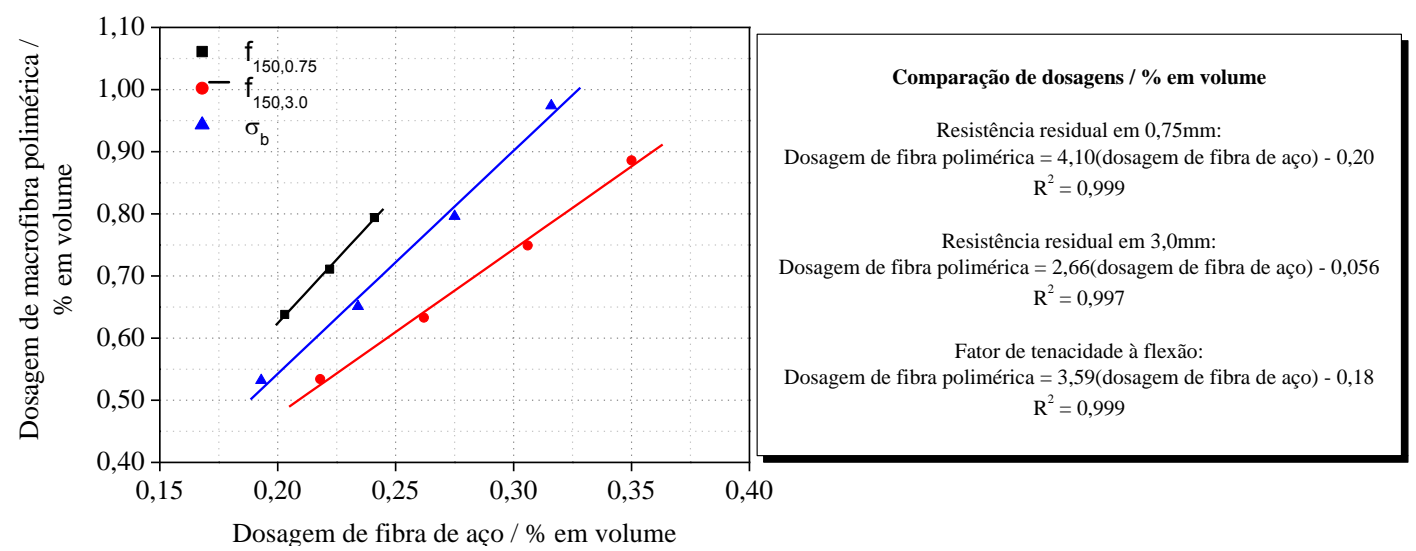

Figura 5: Correlação entre as dosagens de macrofibra e de fibra de aço para demandas de desempenho pré-estabelecidas.

Quando a demanda de desempenho é adotada como o critério de comparação, verifica-se que a macrofibra pode atingir o mesmo desempenho que a fibra de aço. Isso demonstra que a fibra polimérica estudada pode ser uma alternativa confiável na substituição da fibra de aço estudada, desde que respeitadas as equivalências apontadas. 


\section{CONCLUSÕES}

Este trabalho experimental investigou o comportamento de concreto reforçado com uma macrofibra polimérica e com uma fibra de aço por meio de ensaios de flexão utilizando sistema fechado de controle de velocidade de deslocamento. A partir dos resultados obtidos, os seguintes pontos podem ser concluídos:

a) A fibra polimérica estudada pode ser uma alternativa confiável na substituição da fibra de aço estudada, desde que sejam determinados teores com equivalência de desempenho em estudo de dosagem;

b) Dentre as duas fibras ensaiadas, comparando-as em um mesmo teor em volume, verifica-se que as fibras de aço conferem maiores resistências residuais, principalmente para baixos níveis de deformação, devido à sua maior rigidez. Entretanto, para grandes níveis de deformação, a carga resistida pelas fibras poliméricas pode até superar a resistida pelas fibras de aço;

c) Correlacionando as resistências residuais e o fator de tenacidade com as dosagens de fibra, foram obtidos valores de $\mathrm{R}^{2}$ muito altos (na maioria dos casos, superiores a 0,99 ), indicando que as linhas de tendência apresentam excelente aderência aos pontos dispersos nos gráficos;

d) Para as dosagens de fibra normalmente utilizadas em campo, esse tipo de reforço não apresenta influência nas propriedades elásticas do concreto. As fibras aprimoram a resposta pós-fissuração do compósito;

e) Os teores de fibras utilizados não prejudicaram a capacidade de compactação do concreto, devido à utilização de mesa vibratória para o adensamento dos corpos-de-prova. Esse fato é corroborado pela uniformidade dos resultados do teor de ar incorporado e pela baixíssima variação dos resultados de resistência à compressão;

f) A utilização de equipamento com sistema fechado de controle de velocidade de deslocamento (closed-loop) para realização de todos os ensaios conferiu uma grande vantagem ao estudo: o comportamento pós-fissuração medido não foi afetado pela instabilidade pós-pico, ou seja, a resposta do material foi obtida de forma mais precisa e eficaz.

\section{AGRADECIMENTOS}

Os autores agradem à empresa Construquímica pelo suporte na realização dos ensaios.

\section{BIBLIOGRAFIA}

[1] FIGUEIREDO, A.D., "Concreto com fibras", In: Concreto: ciência e tecnologia. 1 ed., cap. 37, Instituto Brasileiro do Concreto (IBRACON), São Paulo, 2011.

[2] BARRAGÁN, B.E., Failure and toughness of steel fiber reinforced concrete under tension and shear, Tese de D.Sc., Universitat Politécnica de Catalunya, Barcelona, Espanha, 2002.

[3] NUNES, N.L., Contribuição para a aplicação do concreto reforçado com fibras de aço em elementos de superfície restringidos, Tese de D.Sc., Escola politécnica, Universidade de São Paulo, São Paulo, Brasil, 2006.

[4] NATARAJA, M.C., et al., "Toughness characterization of steel fiber-reinforced concrete by JSCE approach", Cement and Concrete Research, v. 30, pp. 593-597, 2000.

[5] LIM, D.H., OH, B.H., "Experimental and theoretical investigation on the shear of steel fibre reinforced concrete beams", Engineering Structures, v. 21, pp. 937-944, 1999.

[6] KIM, D.J., NAAMAN, A.E., EL-TAWIL, S., "Comparative flexural behavior of four reinforced cementitious composites", Cement \& Concrete Composites, v. 30, pp. 917-928, 2008.

[7] FIGUEIREDO, A.D., Parâmetros de controle e dosagem do concreto projetado com fibras de aço, Tese de D.Sc., Escola politécnica, Universidade de São Paulo, São Paulo, Brasil, 1997.

[8] BANTHIA, N., SAPPAKITTIPAKORN, M., "Toughness enhancement in steel fiber reinforced concrete through fiber hybridization", Cement and Concrete Research, v. 37, pp. 1366-1372, 2007.

[9] MEHTA, P.K., MONTEIRO, P.J.M., "Evolução em tecnologia do concreto", Concreto, Microestrutura, Propriedades e Materiais, 3 ed., cap. 12, Instituto Brasileiro do Concreto (IBRACON), São Paulo, 2008.

[10] AMERICAN SOCIETY FOR TESTING MATERIALS, ASTM C1609, Standard test method for flexural performance of fiber-reinforce concrete (using beam with third-point loading), $9 \mathrm{p}$, Philadelphia, 2010. 
[11] AMERICAN SOCIETY FOR TESTING MATERIALS, ASTM C1399, Standard test method for obtaining average residual-strength of fiber-reinforced concrete, 6 p, Philadelphia, 2010.

[12] THE JAPAN SOCIETY OF CIVIL ENGINEERS, "JSCE-SF4: Method of tests for flexural strength and flexural toughness of steel fiber reinforced concrete", Concrete library of JSCE. Part III-2 Method of tests for steel fiber reinforced concrete, n. 3, pp. 58-61, 1984.

[13] EUROPEAN COMMITTEE FOR STANDARDIZATION - CEN, EN 14651, Test method for metallic fiber-reinforced concrete - Measuring the flexural tensile strength (limit of proportionality [LOP], residual), 15p, London, 2007.

[14] ASOCIACIÓN ESPAÑOLA DE NORMALIZACIÓN Y CERTIFICACIÓN. UNE 83515, Hormigones con fibras. Determinación de la resistencia a fisuración, tenacidad y resistencia residual a tracción. Ensayo Barcelona. AENOR, 10p., Barcelona, 2010.

[15] GETTU, R., et al., "Testing of concrete under closed-loop control”, Advanced Cement Based Materials, v. 3, n. 2, pp. 54-71, 1996.

[16] BANTHIA, N., DUBEY, A., "Measurement of flexural toughness of fiber-reinforced concrete using a novel technique - Part 1: Assessment and Calibration", ACI Materials Journal, v. 96, n. 6, pp. 651-656, 1999.

[17] BURATTI, N., et al., "Post-cracking behavior of steel and macro-synthetic fibre-reinforced concretes", Construction and Building Materials, v. 25, pp. 2713-2722, 2011.

[18] THE JAPAN SOCIETY OF CIVIL ENGINEERS, JSCE-SF2, "Method of making specimens for strength and toughness tests of steel fiber reinforced concrete", Concrete library of JSCE. Part III-2 Method of tests for steel fiber reinforced concrete, n. 3, pp. 48-55, 1984.

[19] ASSOCIAÇÃO BRASILEIRA DE NORMAS TÉCNICAS, NBR 5739, Concreto: ensaio de compressão de corpos-de-prova cilíndricos, 9p, Rio de Janeiro, 2007.

[20] CECCATO, M.R., NUNES, N.L., FIGUEIREDO, A.D., "Estudo do controle da trabalhabilidade do concreto reforçado com fibras de aço". In: Iv Congresso Iberoamericano de Patologia das Construções e VI Congresso de Controle de Qualidade CONPAT97, Porto Alegre, 1997, v. 2. pp. 539-546, 1997.

[21] ASSOCIAÇÃO BRASILEIRA DE NORMAS TÉCNICAS, NBR 15530, Fibras de aço para concreto Especificação, 7p., Rio de Janeiro, 2007.

[22] ASSOCIAÇÃO BRASILEIRA DE NORMAS TÉCNICAS, NBR NM 67, Concreto: determinação da consistência pelo abatimento do tronco de cone, 8p., Rio de Janeiro, 1998.

[23] ASSOCIAÇÃO BRASILEIRA DE NORMAS TÉCNICAS, NBR 9833, Concreto fresco: determinação da massa específica, do rendimento e do teor de ar pelo método gravimétrico, 7p. Rio de Janeiro, 2008.

[24] ASSOCIAÇÃO BRASILEIRA DE NORMAS TÉCNICAS, NBR NM 47, Concreto: determinação do teor de ar incorporado em concreto fresco - método pressométrico, 23p., Rio de Janeiro, 2005.

[25] ARMELIN, H.S., Contribuição ao estudo do concreto projetado por via seca com fibras de aço destinado ao revestimento de túneis NATM, Dissertação de M.Sc., Escola politécnica, Universidade de São Paulo, São Paulo, Brasil, 1992.

[26] CONCRETE SOCIETY, Technical Report $\mathrm{n}^{\circ} 65$, Guidance on the use of macro-synthetic-fiberreinforced concrete, 76p., The Concrete Society, Camberley, 2007.

[27] di PRISCO, M., et al., "Fiber reinforced concrete in the new FIB model code", In: $3^{\text {rd }}$ fib International Congress, Washington, pp. 1-12, 2010.

[28] BANTHIA, N., DUBEY, A., "Measurement of flexural toughness of fiber-reinforced concrete using a novel technique - Part 2: Performance of Various Composites", ACI Materials Journal, v. 97, n. 1, pp. 3-11, 2000.

[29] ARAKAKI, D.H., FIGUEIREDO, A.D., "Avaliação da tenacidade de concretos reforçados com fibras de aço de alto e baixo teor de carbono", In: IV Simpósio EPUSP sobre Estruturas de Concreto, 2000, São Paulo, 2000.

[30] CONCRETE SOCIETY, Technical Report $\mathrm{n}^{\circ} 63$, Guidance for the design of steel-fiber-reinforced concrete, 92p., The Concrete Society, Camberley, 2007.

[31] MINDESS, S., et al, "Determination of the first-crack strength and flexural toughness of steel fiberreinforced concrete", Advanced Cement Based Materials, v. 1, pp. 201-208, 1994. 
[32] ARMELIN, H.S., BANTHIA, N., "Predicting the flexural post-cracking performance of steel fiberreinforced concrete from the pull-out of single fibers", ACI Materials Journal Detroit, 14p, USA, 1997.

[33] FIGUEIREDO, A.D., NUNES, N.L., TANESI, J., "Mix design analysis on steel fiber reinforced concrete", In: Fifth International RILEM Symposium on Fibre-Reinforced Concretes (FRC), 2000, Lyon, pp. 103-118, 2000. 